\title{
Hearing aid microphones: From electret to piezoelectret transducers
}

\author{
Sessler, Gerhard M. and Hillenbrand, Joachim \\ Technische Universität Darmstadt \\ Merckstrasse 25, D-64283 Darmstadt, Germany
}

\begin{abstract}
Hearing aids are generally equipped with electret microphones. These transducers are of simple design, can be miniaturized, and have good electro-acoustic properties. Recently, a new type of electret microphone, based on piezoelectrets, was introduced. Piezoelectrets consist of a closed-cell foam, often of polypropylene. The foam is poled by a corona discharge and thus assumes piezoelectric properties. Microphones have been built with such piezoelectrets with sensitivities of about $2.5 \mathrm{mV} / \mathrm{Pa}$ which can be increased to $15 \mathrm{mV} / \mathrm{Pa}$ by stacking a number of films. Also, directional microphones based on this principle have been designed. Piezoelectret microphones are of even simpler design than electret microphones and also have good electro-acoustic properties.
\end{abstract}

\section{Introduction}

For several decades, most hearing aids have been equipped with electret condenser microphones (ECMs). Recently, silicon condenser microphones have also been considered for use in hearing aids, but ECMs are still the dominating type. The reasons for the predominance of ECMs are their good electroacoustic properties and that they can be miniaturized [1]. Silicon microphones offer similar properties, although their ENL is somewhat higher. They have the additional advantage that they may be exposed to temperatures up to $260^{\circ} \mathrm{C}$ for short periods of time without degradation. Thus, they can withstand the heat produced during reflow soldering processes and may be used as SMDs on printed circuit boards. Nevertheless, ECMs are still the microphones of choice in present-day hearing aids.

Some years ago, a modified type of electret microphone, utilizing a charged cellular polymer such as polypropylene (PP), was introduced and characterized [2,3]. Since the charged, cellular polymer assumes piezoelectric properties, these transducers are now generally referred to as "piezoelectret" microphones. They also have advantageous properties but are of even simpler construction than the classical ECMs. For this reason, such microphones may be suitable for use in hearing aids. A discussion of their properties, and a comparison with ECMs, is therefore of interest and will be presented in this review. A comparison of piezoelectret microphones with silicon microphones has been given before [4].

\section{Electret microphones}

The schematic design of an ECM is shown in Fig. 1 (left). It consists of an electret which is mostly a polytetrafluoroethylene (PTFE) or polyfluoroethylenepropylene (FEP) film of about $12 \mu \mathrm{m}$ thickness. This film is cemented to a back electrode and then charged by a corona discharge or a similar process to a surface potential of about $200 \mathrm{~V}$. A membrane, often a metallized polymer film, is mounted opposite the electret, but separated from it by a shallow air gap [1]. These microphones can be miniaturized down to dimensions of about 2 to $3 \mathrm{~mm}$. A few typical transducers of this kind are shown in Fig. 1 (right).

The electro-acoustic properties of electret microphones are described by the following data [1]: Their frequency responses are relatively flat in the range extending from $20 \mathrm{~Hz}$ to $20 \mathrm{kHz}$ and they have high sensitivities of about 10 to $20 \mathrm{mV} / \mathrm{Pa}$. Their equivalent noise levels are generally below $25 \mathrm{~dB}(\mathrm{~A})$ and their dynamic range, as determined by nonlinear distortion, extends up to $140 \mathrm{~dB}$ sound pressure level (SPL). The vibration sensitivities are about $-60 \mathrm{dBV} / \mathrm{g}$. They can also be exposed to mechanical and thermal shocks and to humidities in accordance with IEC standards.

\section{Piezoelectret microphones}

Piezoelectrets (also referred to as ferroelectrets) are a new group of charged polymer foams often consisting of cellular polypropylene (PP) [5-8]. A cross section of such a piezoelectret film is shown in the upper part of Fig. 2 (left). For the films to assume piezoelectric properties they have to be electrostatically charged, which is preferably achieved by a corona process, see Fig. 2 (right). The charge distribution so obtained is schematically illustrated in Fig. 2 (left, bottom). The voids with their positive and negative 
charges at the air/polymer-interfaces possess large dipole moments which induce image charges on the metal electrodes. Application of an external force changes the dimension of the voids, which in turn changes their dipole moments and thus the image charges. This is the origin of the piezoelectric activity. An advantage of PP piezoelectrets is that their piezoelectric and mechanical properties can be easily adapted to specific applications by pressure expansion processes, which change the thickness and Young's modulus of the films [9-11]. Further advantages of piezoelectret films are their high piezoelectric $d_{33}$-coefficients, low weight, high flexibility, mechanical stability, availability in large areas, and low material cost [7]
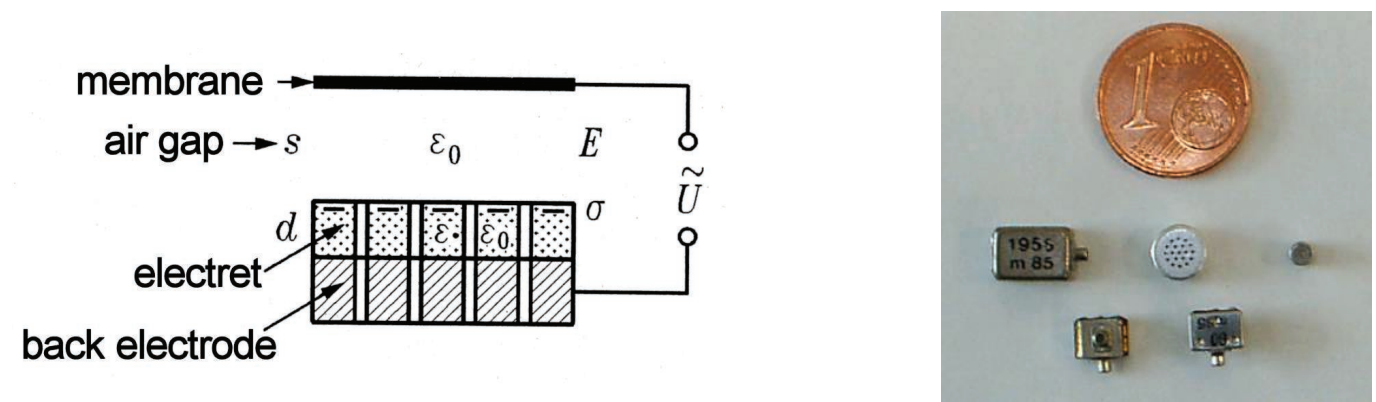

Figure 1: Schematic representation and operating principle of the electret microphone (left) and view of various electret microphones (right).
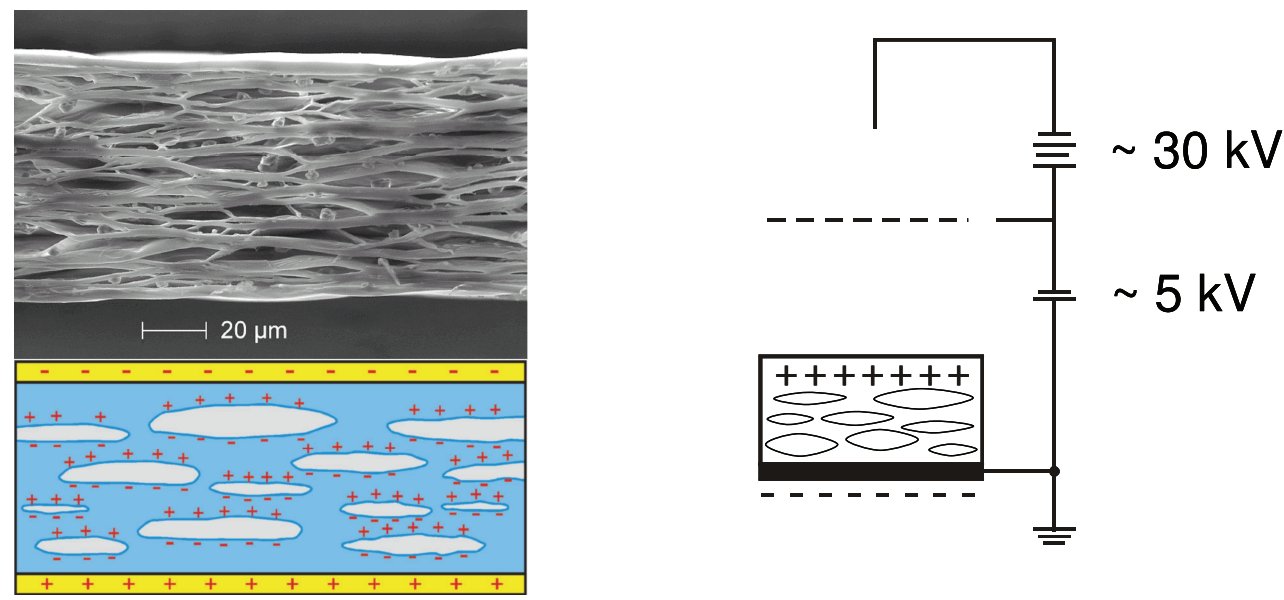

Figure 2: SEM picture and schematic cross section of a piezoelectret film (left) and corona apparatus used for charging the films (right).

Because of their small elastic modulus, piezoelectrets are easily compressed by sound waves and electrical signals are therefore generated upon acoustic excitation. If used as a microphone, an air gap as in conventional electret microphones or an additional air volume is not required. The design of such microphones, schematically illustrated in Fig. 3 , is thus very simple [2,3]. Apart from the piezoelectret film, only provisions for film mounting, shielding, and contacting are required. The sensitivity $M$ of these microphones is given by [12]

$$
M=d_{33} \frac{\left(s_{1}+\varepsilon s_{2}\right)}{\varepsilon \varepsilon_{0}}
$$

where $s_{1}$ and $s_{2}$ are the combined thicknesses of all solid or gas parts of the cellular film, respectively, and $\varepsilon_{0}$ and $\varepsilon$ are the absolute and relative permittivities, respectively. Since $M$ is proportional to $d_{33}$, one expects the sensitivity to drop slightly with frequency, as observed for $d_{33}$ [12]. For a microphone consisting of a stack of $n$ films, connected electrically in series, the open-circuit sensitivity increases proportionally to the number of films and is therefore given by $n M$. This is experimentally confirmed by the results 
depicted in Fig. 4. The figure also shows that all microphones have relatively flat frequency responses and a microphone consisting of a stack of 6 films has a rather high sensitivity of about $15 \mathrm{mV} / \mathrm{Pa}$ [12].

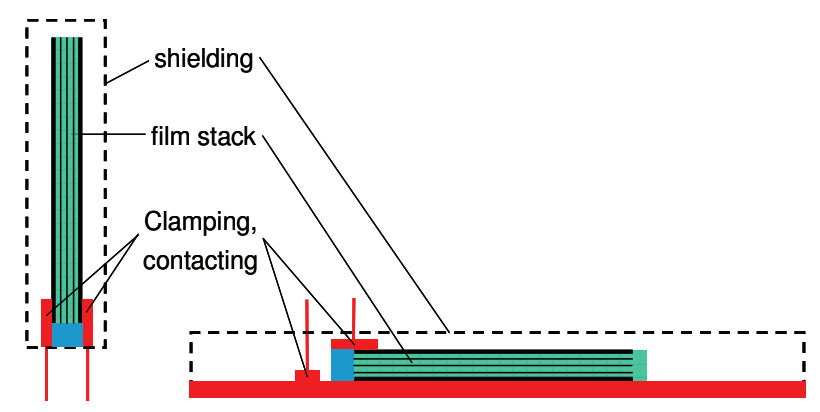

Figure 3: Schematic view of two piezoelectret microphone designs.
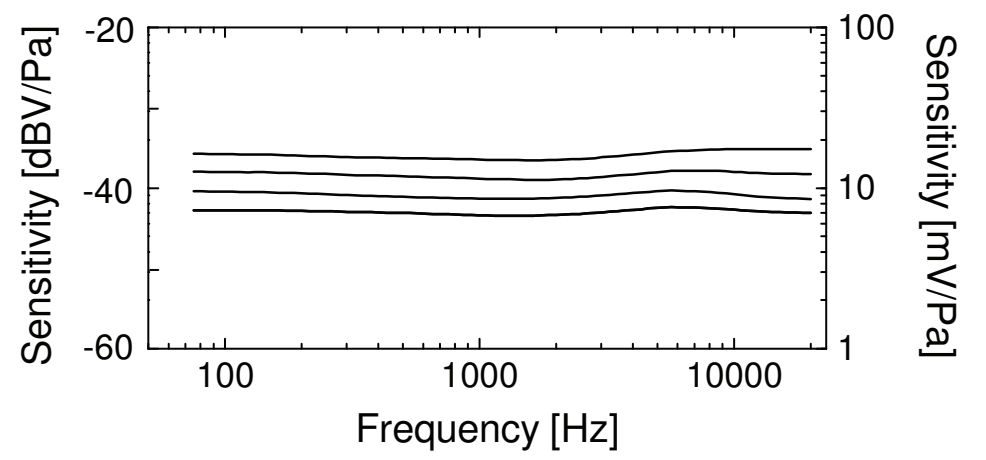

Figure 4: Frequency responses of piezoelectret microphones with stacks of 3 (bottom), 4, 5, and 6 (top) films.

The total capacitance of the film stack decreases inversely proportional to the number of films. Since lower microphone capacitance diminishes the output signal because of capacitive voltage division at the FET input stage, piezoelectret microphones of relatively large area are desirable. To reduce the dimensions of such microphones, coiled film stacks have been used [13,14]. Although a slight reduction of sensitivity due to the coiling process is observed, sensitivities of about $9 \mathrm{mV} / \mathrm{Pa}$ have been achieved with a microphone consisting of a stack of five films.

Another advantage of piezoelectret microphones is their very low total harmonic distortion (THD) [12]. Measurements indicate that the THD increases approximately proportionally to sound pressure and reaches $1 \%$ at $164 \mathrm{~dB}$ SPL. This is lower than the distortion of conventional condenser microphones.

The A-weighted noise spectra of piezoelectret microphones with 1, 2, or 4 piezoelectret films combined with a preamplifier are depicted in Fig. 5. Integrated noise voltages of $2.75,3.4$, and $4.2 \mu \mathrm{V}$, respectively, were measured. This yields A-weighted equivalent noise levels (ENLs) of 35,31 , and $26.5 \mathrm{~dB}(\mathrm{~A})$, respectively [12]. These noise figures correspond closely to the values for the FET preamplifier, as specified by the manufacturer. The improvement of the ENL with the number of films is primarily due to the rise in sensitivity but somewhat reduced by the increase of preamplifier noise with decreasing stack capacitance.

A drawback of the present PP piezoelectret microphones is their thermal instability at temperatures above $50^{\circ} \mathrm{C}$. At these temperatures, the positive and negative charges on the walls of the voids inside the material become mobile and recombine. This problem can best be solved by using film materials with better electret properties, such as the fluoropolymers FEP and PTFE. One approach is to fuse a stack of layers of FEP and PTFE by application of mechanical pressure to the stack at increased temperatures such that minute air bubbles are trapped between the layers [15-17]. After corona charging, one obtains piezoelectrets whose $d_{33}$-coefficients are thermally more stable than those of the PP films, showing only a very small drop at temperatures up to $90^{\circ} \mathrm{C}$ after prior annealing. This method of fabricating piezoelectrets is still in an experimental stage and results are not as consistent as with the PP films. Microphones built with these layered fluoropolymer piezoelectrets have also been tested and they exhibit frequency responses and sensitivities similar to that of PP piezoelectret microphones [18]. 


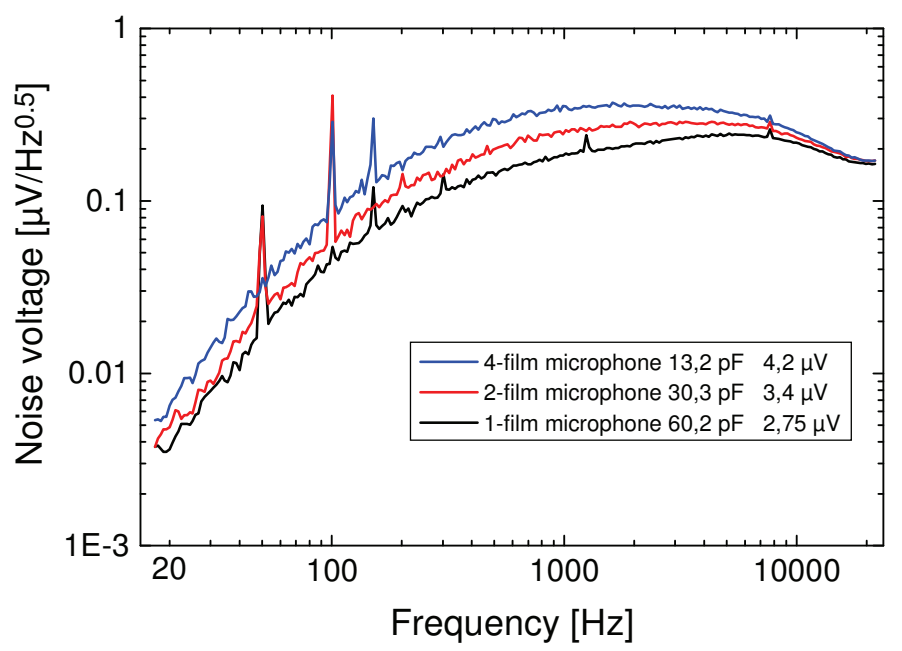

Figure 5: A-weighted noise spectra of omnidirectional piezoelectret microphones with stacks of 1, 2, and 4 PP piezoelectret films.

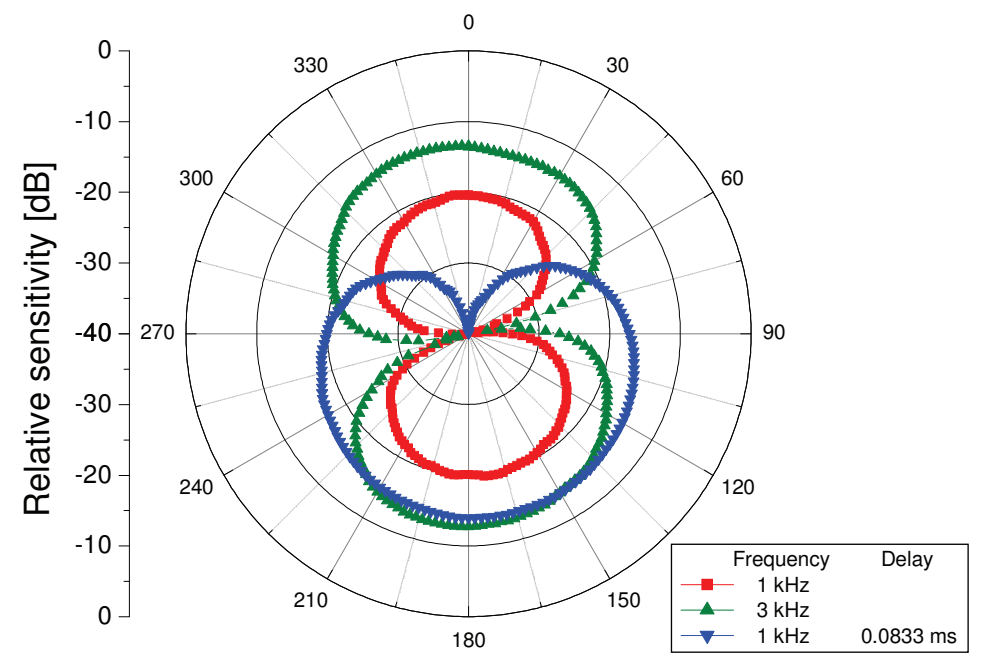

Figure 6: Directivity of a piezoelectret gradient microphone, with and without time delay between the output signals of the two PTFE/FEP piezoelectrets films, measured at different frequencies.

Piezoelectret microphones can also be built as gradient transducers with directional characteristics. This is achieved by placing two units close to each other and combining their outputs in a sum and delay network. For optimal directivity it is necessary to electronically adjust the two units for equal sensitivity. Dipole and cardioid characteristics achieved with such directional microphones, measured in an anechoic chamber, are shown in Fig. $6[4,18]$.

\section{Summary}

ECMs and piezoelectret microphones have both sufficiently good electro-acoustic qualities to be used in hearing aids. In particular, their frequency responses, sensitivities, equivalent noise levels and vibration sensitivities are comparable and satisfactory for this application. Differences exist with respect to highest service temperature and simplicity of design. While ECMs can be used up to $90^{\circ} \mathrm{C}$, the temperature range of the PP piezoelectret microphones is limited to $50^{\circ} \mathrm{C}$. Piezoelectret microphones based on fluoropolymer piezoelectret films have a higher service temperature, but, as pointed out above, are still in an experimental stage and more work is necessary to make them reliable components. As far as simplicity of design is concerned, piezoelectret microphones have two advantages as compared to ECMs: They neither require an air gap nor a back volume. The first fact makes construction of these transducers much simpler while the second fact reduces the bulkiness and volume typical for ECMs. These differences may also be of importance in many other applications where requirements often differ from those for hearing aids. 


\section{Acknowledgments}

The authors gratefully acknowledge financial support by the "Deutsche Forschungsgemeinschaft" and by the "Hessische Ministerium für Wissenschaft und Kunst".

\section{Literature}

[1] R. Lerch, G. Sessler, D. Wolf, "Technische Akustik“, Springer 2009.

[2] R. Kressmann, "New piezoelectric polymer for air-borne and water-borne sound transducers", J. Acoust. Soc. Am. 109, 1412-1416 (2001).

[3] J. Hillenbrand, G. M. Sessler, "High-sensitivity piezoelectric microphones based on stacked polymer films", J. Acoust. Soc. Amer. 116, 3267-3270 (2004).

[4] G. M. Sessler and J. Hillenbrand, "Novel silicon and polymer sensors in acoustics", SENSOR+TEST conference 2009 - SENSOR 2009 Proceedings I, pp. 9-14 (2009).

[5] J. Lekkala, R. Poramo, K. Nyholm, and T. Kaikkonen, "EMF force sensor - a flexible electret film for physiological applications", Med. Biol. Eng. Comput. 34, 67-68 (1996).

[6] G. M. Sessler and J. Hillenbrand, "Electromechanical response of cellular electret films", Appl. Phys. Lett. 75, 3405-3407 (1999).

[7] S. Bauer, R. Gerhard-Multhaupt, and G. M. Sessler, "Ferroelectrets: Soft electroactive foams for transducers", Physics Today 57(2), 37-43 (2004).

[8] J. Hillenbrand and G.M. Sessler, "DC-biased ferroelectrets with large piezoelectric $d_{33}$-coefficients", J. Appl. Phys. 103, 074103 (7p), (2008).

[9] M. Paajanen, H. Minkkinen, and J. Raukola, "Gas diffusion expansion-increased thickness and enhanced electromechanical response of cellular polymer electret films", in Proc. $11^{\text {th }}$ Internat. Sympos. Electrets, pp. 191-194 (2002).

[10] M. Wegener, W. Wirges, R. Gerhard-Multhaupt, M. Dansachmüller, R. Schwödiauer, S. BauerGogonea, S. Bauer, M. Paajanen, H. Minkkinen, and J. Raukola, "Controlled inflation of voids in cellular polymer ferroelectrets: Optimizing electromechanical transducer properties“, Appl. Phys. Lett. 84, 392-394 (2004).

[11] X. Zhang, J. Hillenbrand, and G. M. Sessler, "Improvement of piezoelectric activity of cellular polymers using a double-expansion process", J. Phys. D: Appl. Phys. 37, 2146-2150 (2004).

[12] J. Hillenbrand and G.M. Sessler, "Stacked piezoelectret microphones of simple design and high sensitivity", IEEE Trans. Dielectr. Electric. Insulat. 13, 973-978 (2006).

[13] J. Hillenbrand, P. Pondrom, and G. M. Sessler, "Piezoelectret-microphones with high capacitance and sensitivity", in Proc. of the $19^{\text {th }}$ Internat. Congress on Acoustics (Madrid, 2007).

[14] J. Hillenbrand, P. Pondrom, and G. M. Sessler, "Piezoelektret-Mikrofone mit nicht-planaren Folienstapeln und hoher Kapazität", DAGA 2008, pp. 425-426 (2008).

[15] X. Zhang, J. Hillenbrand, and G. M. Sessler, "Ferroelectrets with improved thermal stability made from fused fluorocarbon layers", J. Appl. Phys. 101, 054114 (8p) (2007).

[16] Z. Hu, H. von Seggern, "Breakdown-induced polarization buildup in porous fluoropolymer sandwiches: a thermally stable piezoelectret”, J. Appl Phys. 99, 024102 (5p), (2006).

[17] R. A. P. Altafim, X. Qiu, W. Wirges, R. Gerhard, R. A. C. Altafim, H. C. Basso, W. Jenninger, and J. Wagner, "Template-based fluoroethylenepropylene piezoelectrets with tubular channels for transducer applications", J. Appl. Phys. 106, 014106 (5p), (2009).

[18] J. Hillenbrand, X. Zhang, and G. M. Sessler, "Piezoelektret-Mikrofone mit Fluoropolymerfolien und verbesserter Temperaturstabilität", in Fortschritte der Akustik, DAGA 2007, pp. 495-496 (Deutsche Gesellschaft für Akustik e. V., 2007). 